\title{
Polycystic Ovarian Syndrome - A Pain for Young Women? An Autobiographical Case Studies
}

\author{
Jyotsna Gupta $^{1 *}$ and Suresh Kishanrao ${ }^{2}$ \\ ${ }^{1}$ Bachelor's in Occupational Therapy (BOT) (MPH), Karnataka State Rural \\ Development and Panchayat Raj University, Gadag, Karnataka, India \\ ${ }^{2}$ Public Health Consultant and Visiting Professor, MPH, Karnataka State Rural \\ Development and Panchayat Raj University, Gadag, Karnataka, India \\ *Corresponding Author: Jyotsna Gupta, Bachelor's in Occupational Therapy (BOT) \\ (MPH), Karnataka State Rural Development and Panchayat Raj University, Gadag, \\ Karnataka, India.
}

Received: December 27, 2021

Published: January 31, 2022

(C) All rights are reserved by Jyotsna

Gupta and Suresh Kishanrao.

\section{Abstract}

Polycystic ovary syndrome (PCOS) is a common endocrine disorder predominantly affecting young women of reproductive age. Current Diagnostic Criteria as per NIH, USA International PCOS Guidelines 2018, 2 of 3 ensuing criteria are needed i) Hyperandrogenism 2) Oligo-and/or anovulation 3) Polycystic ovaries. In 1990 NIH recommended presence of both Hyperandrogenism and Chronic Anovulation. Changes in the diagnostic criteria greatly affect the prevalence of PCOS. Prevalence rates have been reported as low as $1.6 \%$ using a combination of all three criteria and as high as $18 \%$ in USA [1]. Studies of PCOS in India carried out in convenience samples reported a prevalence of $3.7 \%$ to $22.5 \%$, with $9.13 \%$ to $36 \%$ prevalence in adolescents only. World Health Organization (WHO) estimates that PCOS had affected 3.4\% of women worldwide in 2012. Globally, prevalence estimates of PCOS are highly variable, ranging from $2.2 \%$ to as high as $26 \%$.

The condition is often misdiagnosed and poorly understood, is a lifelong disorder that can be managed with appropriate medical care. Most common symptoms are Missed periods, excess body hair including the face (hirsutism) and Weight gain in especially around the abdomen, more Acne during the periods, baldness, or thinning hair occurs and Infertility occurs if not treated for long. Hormonal assessment and Ultrasonography that shows large ovaries and multiple cysts in the ovary clinch the diagnosis. The management protocol depends upon hormonal therapy and monitoring associated metabolic syndromes like weight gain, Diabetes, Obesity and Cardiovascular symptoms. We present an autobiographical case of myself, a 25-year girl now, presenting the current and third episode of PCOS. I consulted a gynecologist at a private hospital in GADAG, Karnataka, 582101, with the complaints of white discharge, backache, hirsutism and hair fall, acne, feeling stressed apart from irregular periods and adding fat around my waist since 13 JANUARY 2021. She on clinical examination found breasts normal but with darkening of areola and nipple, a few hairs, and some milk like fluid oozing on squeezing the nipple. My blood pressure was $130 \backslash 90 \mathrm{~mm} \mathrm{hg}$, pulse rate $-102 /$ minute, RR - 20/ minute, weight $63 \mathrm{~kg}$, height - $155.4 \mathrm{~cm}$. Based on increased testosterone, low FSH, elevated LH, elevated estrogens, increased prolactin, and USC showing bilateral enlarged ovaries with multiple cysts with more than 15 follicles, I was diagnosed as a case of PCOS and put on hormonal therapy. I was first put on oral contraceptive pill for 3 months and then on Myo-Inositol (Myo Inositol, D-Chiro Inositol with Folic Acid) one tablet twice a day for 3 months, once a day for the last 2 months. All signs have disappeared now except for periodical episodes of headache and my menstrual cycles are normal for last 2 months.

Keywords: Missing or Delayed Monthly Periods; Hirsutism; Weight Gain; Acne, and Central Alopecia; Hormonal Assays; Ultrasonography; Oligomenorrhoea; Fear of Infertility; Depression 


\section{Introduction}

Polycystic ovarian syndrome (PCOS) is a common multifaceted disorder found among females of the reproductive age presenting major clinical conditions of hirsutism, oligomenorrhea and infertility. Socioeconomic studies from India have observed PCOS as a lifestyle disorder highly prevalent among middle and high-income urban population as compared to rural population. It is a syndrome or group of symptoms three main features being cysts in the ovaries, high level of male hormones, irregular or skipped periods. The clinical manifestations include heavy bleeding, severe abdominal pain during periods, weakness, mood changes such as irritated feeling and feeling stress before 2-3 days of periods, hair growth, acne, weight gain, darkening of the skin, headaches and infertility and increased risk of metabolic diseases such as high blood sugar, high blood pressure, low HDL (good cholesterol), high LDL (bad cholesterol) together these factors are called metabolic syndrome i.e., heart disease, diabetes stroke etc. besides psychological dysfunction (depression), severity causes Endometrial cancer.

Diagnostic Criteria: According to the Diagnostic Criteria of NIH, USA International PCOS Guidelines 2018, 2 of the ensuing 3 criteria are needed i) Hyperandrogenism 2) Oligo-and/or anovulation 3) Polycystic ovaries. In 1990 NIH had recommended presence of both Hyperandrogenism and Chronic Anovulation. Changes in the diagnostic criteria greatly affect the prevalence of PCOS.

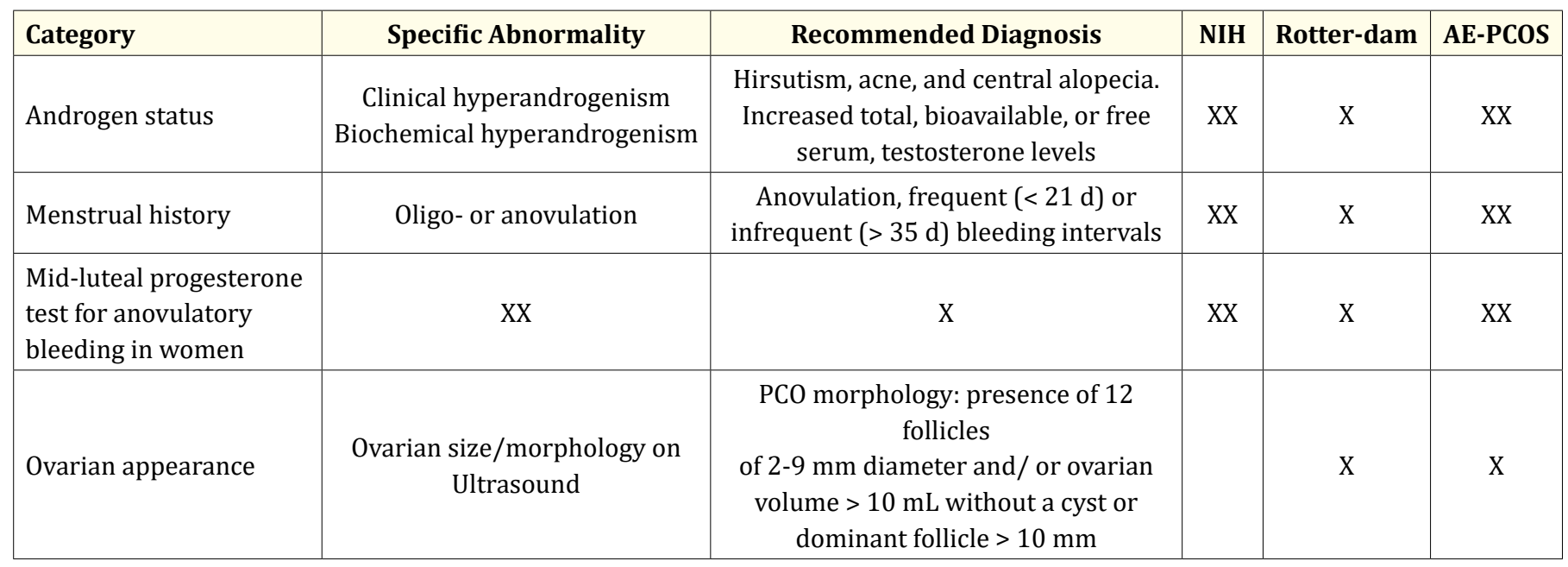

Table 1: Diagnostic criteria for PCOS.

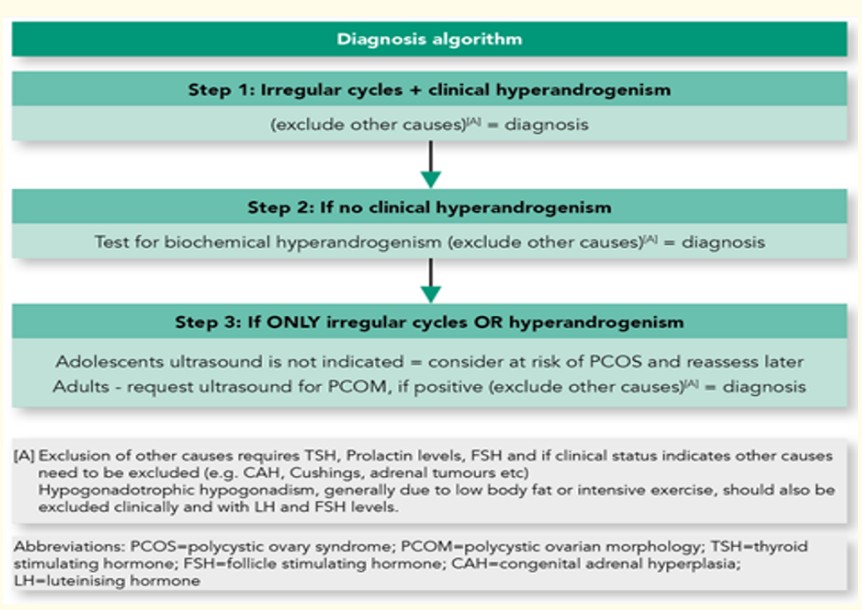

Figure a: Management of polycystic ovary syndrome Guidelines. co.uk.
Magnitude of the problem

A study that reviewed the incidence and DALYs during 2007 and 2017 in 2017, indicated that the total number of disabilityadjusted life-years (DALYs) due to PCOS among women of reproductive age was 0.43 million worldwide, a $12.08 \%$ increase between 2007 to 2017. The global age-standardized DALY rate due to PCOS among reproductive age women was 21.96 per 100,000 population in 2017, an increase of $1.91 \%$ from 2007 to 2017 . The age standardized rate (per 100000 ) for polycystic ovary syndrome among women of reproductive age (15-49 years) in South Asia at increased from 147258.67 in 2007 to 151685.88 in 2017 an increase of 3.01\%. The DALY increased from 86.88 in 2007 to 87.93 in 2017 an increase of 1.21 over a decade [1]. 
Polycystic ovary syndrome (PCOS) is a health problem that affects from 3.7 to 22.5 \% Indian women, whereas in USA it affects 1 in 10 women of childbearing age. Prevalence rates have been reported as low as $1.6 \%$ using a combination of all three criteria and as high as $18 \%$ in USA $[2,3]$. PCOS is also a common and treatable cause of infertility. In 2017, 1.55 million incident cases of PCOS among women of reproductive age (15-49 years) were reported globally, representing an increase of $4.47 \%$ (2.86-6.37\%) from 2007 to 2017. The global age-standardized incidence rate of PCOS among women of reproductive age was 82.44 per 100000 population in 2017, an increase of $1.45 \%$ from 2007 to 2017.

Prevalence of PCOS India ranges from 3.7 to $22.5 \%$ percent depending on the population studied and the criteria used for diagnosis. Abnormalities in leptin - adiponectin (adipocyte biology), oxidative stress and autoimmunity are among the mechanisms studied regarding pathogenesis of PCOS. PCOS is a multifaceted disease with a spectrum of manifestations affecting not only women of childbearing age, but also adolescents and postmenopausal women. PCOS by the nature of the disease, adversely influences the fertility and reproductive health of the affected women; moreover, with its association with other lifestyle diseases, it is also the cause of significant cardiovascular and metabolic morbidity. The exact etiology and pathogenesis of PCOS are still an area of active research, although multiple hypothesis has been postulated, ranging from generic susceptibility to environment exposure, both in utero and in the postnatal life.

There are four types of PCOS described i) Insulin-resistant PCOS- the most common type, caused by smoking, sugar, pollution and trans-fat. In this, high levels of insulin prevent ovulation and trigger the ovaries to create testosterone. ii) Pill-induced PCOS the second most common PCOS, caused using birth control pills which suppress ovulation. iii) INFLAMMATORY PCOS -Inflammation is caused due to stress, toxins of environment and inflammatory dietary like gluten. and iv) HIDDEN PCOS-a simpler form of PCOS, caused due to Thyroid disease, deficiency of iodine (ovaries need iodine), vegetarian diet (zinc deficient needed to ovaries) and artificial sweeteners [5].

I diagnosed as an case of inflammatory type of PCOS.

\section{Case Presentation}

Aged about 25 years now, I was a 22-year college going girl when first consulted a gynecologist in medical college Varanasi with the complaints of irregular menses or prolonged cycle, abnormal weight gain, acne, hirsutism, headache, anxiety, mood swings and feeling stressed before periods, hair fall for over 6 months. I had my menarche at 14 years. Initial monthly periods were regular, but after 2 years I observed that my periods getting irregular but did not worry much till the age of 17 years. Then, after I missed my periods for 6 months, I consulted a gynecologist in Banaras hospital. After taking detailed history, physical examination, and ultrasonography, I was diagnosed having PCOS. I was given some hormonal medicines and advised for taking healthy food. After taking medicines for 3 months my periods became normal, and I continued the same till was 22 years old. They I stopped taking medication, unfortunately after 1 year my periods got getting irregular while I was working in Hyderabad as an occupational therapist. Hence, I consulted a gynecologist in Hyderabad who prescribed contraceptive pills for 3 months resulting in normal periods until I was 24 years old. I had moved to Gadag for doing my master's in public health (MPH) and at the age of 25 I again started getting irregularity in periods. This time I had whitish discharge, backache, hirsutism and hair fall, acne, feeling stressed apart from irregular periods. Now I consulted a gynecologist at a private hospital in Gadag, Karnataka 582101.

On clinical examination breasts were normal abnormal darkening of areola and nipple, some abnormal milk like fluid was seen on squeezing the nipple, some hairs present on breast and nipples. Hirsutism and Weight gain around my waist was also noted. On examination my blood pressure was $-130 \backslash 90 \mathrm{~mm} \mathrm{hg}$, pulse rate - 102/minute, RR - 20/minute. weight - $63 \mathrm{~kg}$, height - $155.4 \mathrm{~cm}$.

\section{Investigations}

My blood hormonal report showed an increased testosterone, low FSH, elevated LH, elevated estrogens, increased prolactin no thyroid abnormality, and blood sugar was within normal range. USC showed enlarged ovary with multiple ovarian cysts and characteristically have more than 15 follicles per ovary seen. cysts in right ovary and I was diagnosed as a case of Inflammatory type of PCOD based on all these investigation. 
Sonography showing ovarian cysts

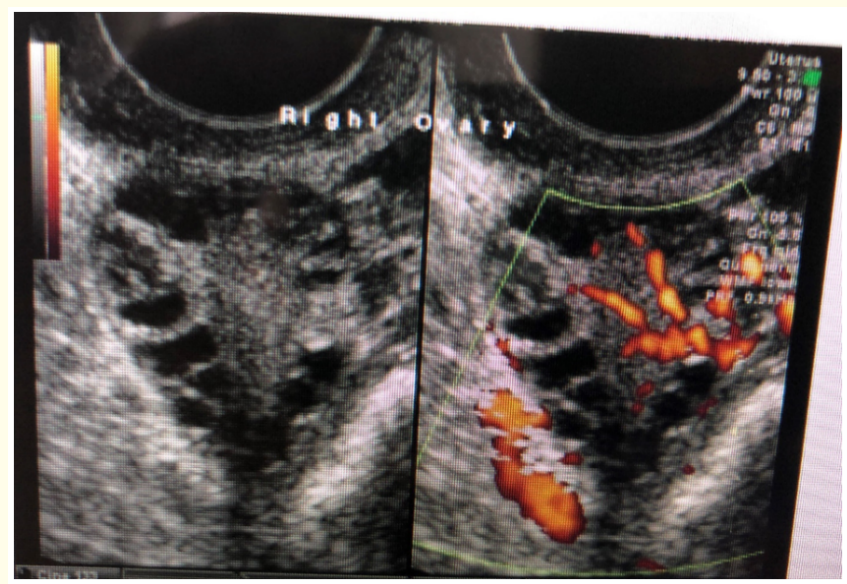

Figure b

\section{Treatment}

I was first put on first oral contraceptive pill for 3 months for inducing predictable cyclic menses and for reducing LSH and lower ovarian androgen production. Then I was put on hormonal therapy myoinositol for regulating my hormonal imbalance such as follicle -stimulating hormone which improves my menstrual cycle, clomiphene, letrozole to induces ovulation for 3 months. I have been consulting regularly every 3 months and SOS when any symptoms recurred. I was advised for a stress-free living, healthy lifestyle of doing exercise, yoga and meditation, avoid of milk products, and to eat more green vegetables and fruits in diet. I was advised for myoinositol twice a day and for 2 months after the symptoms reduced the doses has been reduced to 1 time a day.

\section{Follow-up}

After taking medication in Gadag for 2 months the blood pressure became normal i.e., $120 \backslash 80$ and the menstrual cycle also returned to normal of 28-day cycle. The weight reduced by $2 \mathrm{kgs}$ in one month after taking treatment i.e., $61 \mathrm{~kg}$. the facial hair also showed signs of reduction after 15 days. I continue to have episodes of headache. I continue to be on medication with a reducing dose in $7^{\text {th }}$ month.

\section{Pathophysiology}

PCOS is a disease with a complex multipronged pathogenesis, yet to be understood fully. However, a few risk factors have been associated including a) BMI as an important indicative and more so the changes in BMI during adolescence are positively associated (as measured by changes in waist circumference) with a history of weight gain, b) higher energy intake and glycemic index, low physical activity, smoking, and alcohol intake all linked to increasing BMI. c) Significantly higher Insulin Resistance (fasting serum insulin) with normal oral glucose tolerance test d) primary risk factor of chronic anovulation leading to menstrual cycle disturbances d) An association between early menarche e) Cutaneous manifestations like early or persistent or frequent relapsing acne, hirsutism for $>$ two years, associated with PCOS.

The various pathogenetic mechanisms of PCOS include abnormal gonadotropin-releasing hormone $(\mathrm{GnRH})$ regulation leading to increased luteinizing hormone (LH) and decreased FSH; decreased response of ovarian follicles to FSH; increased anti-Mullerian hormone (AMH); follicular arrest and increased secretion of testosterone, estradiol and dehydroepiandrosterone (DHEA). Obesity, especially abdominal fat deposition, is the major predisposing factor for the expression of IR and metabolic phenotype in PCOS Thus, IR at post-receptor level and adipocyte dysfunction are responsible for the metabolic consequences of PCOS [10].

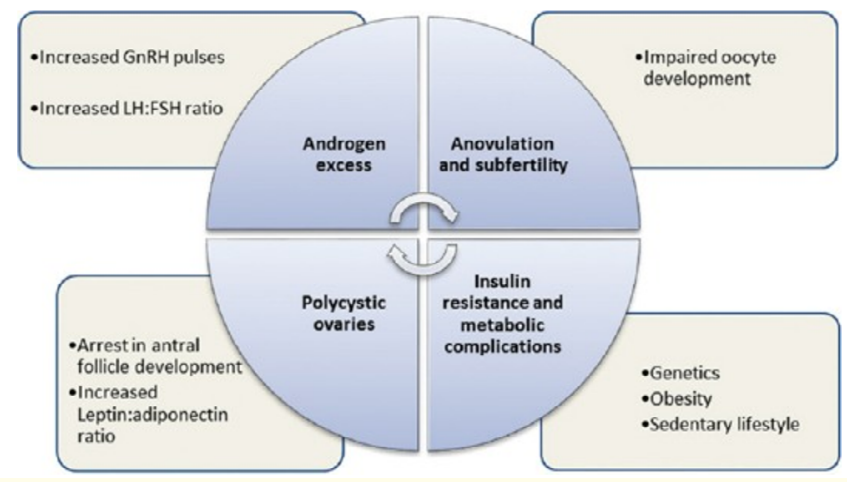

Figure c: Concept of pathophysiology of polycystic ovary syndrome. LH, Luteinizing Hormone; FSH, Follicle-stimulating Hormone; GnRH, Gonadotropin-Releasing Hormone.

\section{Genetics}

PCOS may be genetically determined came from some twin studies, but I do not have such an history of such incidences in the previous two generation. 


\section{Social issues}

Facial hair, increases weight and other cosmetic changes lead to rejection of female leading to psychological issues anxiety and depression, there are also possibilities of infertility leading to familial and societal neglect. Such cases may remain hidden or unreported for long time due to poverty, fear, feeling shy, social, and cultural barriers, lack of proper health facilities and timely diagnosis., acceptance of the condition as a curse may lead to psychosocial problems.

\section{Indication for surgery}

Very rare, but in this case, there is no requirement of surgery.

\section{Discussion}

There are only a few studies of PCOS in India and most of them are on convenience-based sampling, which might not reflect the true status of PCOS prevalence in the community.

The closest study comparable to my case is from Lucknow, in which college-going women with menstrual irregularity and hirsutism, in the age range of 18-25 year were studied. It had reported a calculated prevalence using the NIH criteria, to be $3.7 \%$ [4].

A study conducted in an urban community of Mumbai, just like me an urban educated girl found that the prevalence of PCOS was 22.5 per cent by the Rotterdam criteria and 10.7 per cent by the Androgen Excess Society criteria [6].

A study conducted among medical students at a private medical college in south India using the modified Cronin questionnaire of 10 items, found that PCOS was a common disorder among the participants and reported a high incidence of mood disorders among as is in my case of frequent mood changes.

A study from Andhra Pradesh studied young women from a residential college and found that 9.13 per cent of them satisfied the Rotterdam criteria for PCOS.

Vidya Bharathi., et al. showed that the prevalence of PCOS diagnosed by the Rotterdam criteria in community-dwelling women from rural and urban areas of Chennai was $6 \%$.

Hence, it can be noticed that the prevalence of PCOS in India ranges from 3.7 to 22.5 per cent [9].

\section{Conclusion}

Here I shared my experience and challenges in my collage days how I suffered and faced problems and how I noticed and consulted with the gynecologist. At the age of 22 I faced a problem of irregular menses, abnormal weight gain, heavy bleeding, hair fall, darkening of the skin, headache, acne, mood swings, depression. After that I consulted a gynecologist by doing some investigation and ultrasonography I diagnosed a case of PCOD. After That I was treated for PCOS by hormonal therapy for 3 months and showed great signs of improvement in most of the symptoms. After taking medications and preventive measures my menstrual cycle returns to normal. By taking Timely intervention and proper treatment which was supported by my conscious family my challenges become less. Mainly in rural areas adolescent girls face the same problems but due to shyness they are unable to tell their families, So, its important to do resolution of the clinical problems that neglect and delay due to psychosocial barrier would have led to long term medical and mental health issues.

\section{Take home messages}

- Polycystic ovary syndrome (PCOS) is a common endocrine lifelong disorder predominantly affecting women of reproductive age starting around early 20 years with diverse Clinical manifestations.

- It's one of the causes of preventable infertility

- $\quad$ Often misdiagnosed and poorly understood, polycystic ovary syndrome (PCOS) can be managed with appropriate medical care or surgical methods, Further research is needed to sharpen our therapeutic armamentarium.

\section{Bibliography}

1. Jingjing Liu., et al. "Measuring the global disease burden of polycystic ovary syndrome in 194 countries: Global Burden of Disease Study 2017". Human Reproduction 36.4 (2021): 11081119.

2. Wendy M Wolf., et al. "Geographical Prevalence of Polycystic Ovary Syndrome as Determined by Region and Race/Ethnicity". International Journal of Environmental Research and Public Health 15.11 (2018): 2589.

3. Mohammad Ashraf Ganie., et al. "Epidemiology, pathogenesis, genetics \& management of polycystic ovary syndrome in India". Indian Journal of Medical Research 150.4 (2019): 333-344. 
4. Gill H., et al. "Prevalence of polycystic ovary syndrome in young women from North India: A community-based study". Indian Journal of Endocrinology and Metabolism 16 (2012): S389-392.

5. Sonia Malik., et al. "Management of Polycystic Ovary Syndrome in India". President, Indian Fertility Society (2015): 182.69.98.25.

6. Roshan Dadachanji., et al. "TYPES OF PCOS”. (2021).

7. Beena Joshi., et al. "A cross-sectional study of polycystic ovarian syndrome among adolescent and young girls in Mumbai, India". Indian Journal of Endocrinology and Metabolism 18.3 (2014): 317-324.

8. Ritu Deswal., et al. "The Prevalence of Polycystic Ovary Syndrome: A Brief Systematic Review". Journal of Human Reproductive Sciences 13.4 (2020): 261-271.

9. Nitin Joseph., et al. "Study on the proportion and determinants of polycystic ovarian syndrome among health sciences students in South India". Journal of Natural Science, Biology, and Medicine 7.2 (2016): 166-172.

10. Nidhi R., et al. "Prevalence of polycystic ovarian syndrome in Indian adolescents". Journal of Pediatric and Adolescent Gynecology 24 (2011): 223.

\section{Assets from publication with us}

- Prompt Acknowledgement after receiving the article

- Thorough Double blinded peer review

- Rapid Publication

- Issue of Publication Certificate

- High visibility of your Published work

Website: www.actascientific.com/

Submit Article: www.actascientific.com/submission.php

Email us: editor@actascientific.com

Contact us: +919182824667 